\title{
Potential Shaping and the Method of Controlled Lagrangians
}

\author{
Anthony M. Bloch ${ }^{1}$ \\ Department of Mathematics \\ University of Michigan \\ Ann Arbor, MI 48109 \\ abloch@math.lsa.umich.edu
}

\author{
Naomi Ehrich Leonard ${ }^{2}$ \\ Dept. of Mechanical and Aerospace Engineering \\ Princeton University \\ Princeton, NJ 08544 \\ naomi@princeton.edu
}

\author{
Jerrold E. Marsden ${ }^{3}$ \\ Control and Dynamical Systems \\ California Institute of Technology 107-81 \\ Pasadena, CA 91125 \\ marsden@cds.caltech.edu
}

\begin{abstract}
We extend the method of controlled Lagrangians to include potential shaping for complete state-space stabilization of mechanical systems. The method of controlled Lagrangians deals with mechanical systems with symmetry and provides symmetry-preserving kinetic shaping and feedback-controlled dissipation for state-space stabilization in all but the symmetry variables. Potential shaping complements the kinetic shaping by breaking symmetry and stabilizing the remaining state variables. The approach also extends the method of controlled Lagrangians to include a class of mechanical systems without symmetry such as the inverted pendulum on a cart that travels along an incline.
\end{abstract}

\section{Introduction}

We introduce potential shaping into the method of controlled Lagrangians, our constructive approach to the derivation of stabilizing control laws for Lagrangian mechanical systems. This allows us to achieve complete state-space stabilization for underactuated systems. This class of mechanical systems we address tends to be difficult to control; for example, the systems are typically not feedback linearizable.

The guiding principle behind our method of controlled Lagrangians is to consider a class of control laws that yield closed-loop dynamics which remain in Lagrangian form. The method thus provides a natural class of energy-based Lyapunov functions and yields

\footnotetext{
${ }^{1}$ Research partially supported by the National Science Foundation grant DMS-9803181 and AFOSR grant F49620-96-1-0100

${ }^{2}$ Research partially supported by the National Science Foundation under grant BES-9502477 and by the Office of Naval Research under grant N00014-98-1-0649.

${ }^{3}$ Research partially supported by AFOSR Grant F49620-951-0419
}

$0-7803-5250-5 / 99 / \$ 10.00$ (c) 1999 IEEE large and computable basins of stability which become asymptotically stable when dissipative controls are added.

We give in Bloch, Leonard and Marsden [1997,1998,1998a,1999b] sufficient conditions under which our algorithmic approach provides a control law that yields a closed-loop system in Lagrangian form. These sufficient conditions we refer to as matching conditions since they ensure that the Euler-Lagrange equations derived from the controlled Lagrangian are consistent with available control inputs, i.e., they match the controlled Euler-Lagrange equations for the given mechanical system.

The systems considered in Bloch, Leonard and Marsden [1997,1998,1999a,1999b] are mechanical systems with symmetry and the Lagrangian for the closedloop system (the controlled Lagrangian) is the Lagrangian for the uncontrolled system with a reshaped kinetic energy which retains the original symmetry. We added feedback-controlled dissipation and proved asymptotic stabilization in all state variables except for the symmetry group variables. For example, in the case of the inverted pendulum on the cart, we drive the pendulum to the upright position and the cart to rest but not necessarily positioned at the origin.

In this paper we complete the strategy by augmenting the construction to include symmetry-breaking modifications to the potential energy. This provides the means to stabilize all state variables; for instance, in the cart-pendulum example, the cart position can be driven to the origin as well.

We also extend the class of mechanical systems considered to include those with original potential energy that breaks symmetry. For example, the extended class of systems includes the inverted pendulum on a cart that travels on an incline.

The method of controlled Lagrangians has its ori- 
gins in Bloch, Krishnaprasad, Marsden and Sánchez de Alvarez [1992] and Bloch, Marsden and Sánchez de Alvarez [1997]. Our shaping of potential energy is done in the spirit of van der Schaft [1986] and Leonard [1997]. Auckly, Kapitanski and White [1998] and Hamberg [1999] present related work on matching and asymptotic stabilization. Earlier relevant work on energy methods in control and stabilization includes Wang and Krishnaprasad [1992], Baillieul [1993], and Åström and Furuta [1996].

In $\S 2$ we outline the controlled Lagrangian approach to stabilization and review matching and stabilization by kinetic shaping. In $\S 3$ we introduce potential shaping and present sufficient conditions for matching. In $\S 4$ we provide sufficient conditions and the construction for complete state-space stabilization. In $\S 5$ we apply the construction to the inverted pendulum on a cart that travels on an incline.

\section{Method of Controlled Lagrangians}

In this section we review the controlled Lagrangian approach to (partial state-space) stabilization by kinetic shaping as presented in Bloch, Leonard and Marsden $[1998,1999 a, 1999 b]$. The approach begins with a mechanical system with an uncontrolled (free) Lagrangian equal to kinetic energy minus potential energy. We modify the kinetic energy to produce a new controlled Lagrangian which describes the dynamics of the controlled closed-loop system.

Suppose our system has configuration space $Q$ and that a Lie group $G$ acts freely and properly on $Q$. It is useful to keep in mind the case in which $Q=S \times G$ with $G$ acting only on the second factor by acting on the left by group multiplication. For example, for the inverted planar pendulum on a cart, $Q=S^{1} \times \mathbb{R}$ with $G=$ $\mathbb{R}$, the group of reals under addition (corresponding to translations of the cart).

The goal of kinetic shaping is to control the variables lying in the shape space $Q / G$ using controls that act directly on the variables lying in $G$. Assume that the Lagrangian is invariant under the action of $G$ on $Q$, where the action is on the factor $G$ alone. In many examples the invariance amounts to the Lagrangian being cyclic in the $G$-variables. Accordingly, this produces a conservation law for the free system. The construction preserves the invariance of the Lagrangian, thus providing a modified or controlled conservation law. Throughout this paper we will assume that $G$ is an abelian group.

The essence of the modification of the Lagrangian involves changing the metric tensor $g(\cdot, \cdot)$ that defines the kinetic energy $\frac{1}{2} g(\dot{q}, \dot{q})$. The tangent space to $Q$ can be split into a sum of horizontal and vertical parts defined as follows: for each tangent vector $v_{q}$ to $Q$ at a point $q \in Q$, we can write a unique decomposition $v_{q}=$ Hor $v_{q}+\operatorname{Ver} v_{q}$, such that the vertical part is tangent to the orbits of the $G$-action and where the horizontal part is the metric orthogonal to the vertical space; that is, it is uniquely defined by requiring the identity

$$
g\left(v_{q}, w_{q}\right)=g\left(\operatorname{Hor} v_{q}, \text { Hor } w_{q}\right)+g\left(\operatorname{Ver} v_{q}, \operatorname{Ver} w_{q}\right)
$$

where $v_{q}$ and $w_{q}$ are arbitrary tangent vectors to $Q$ at the point $q \in Q$.

For the kinetic energy of our controlled Lagrangian, we use a modified version of the right hand side of equation (2.1). The potential energy remains unchanged. The modification consists of three ingredients:

1. a new choice of horizontal space, denoted $\mathrm{Hor}_{\tau}$,

2. a change $g \rightarrow g_{\sigma}$ of the metric on horizontal vectors and

3. a change $g \rightarrow g_{\rho}$ of the metric on vertical vectors.

Let $\xi_{Q}$ denote the infinitesimal generator corresponding to a Lie algebra element $\xi \in \mathfrak{g}$, where $\mathfrak{g}$ is the Lie algebra of $G$ (see Marsden [1992] or Marsden and Ratiu [1994]). Thus, for each $\xi \in \mathfrak{g}, \xi_{Q}$ is a vector field on the configuration manifold $Q$ and its value at a point $q \in Q$ is denoted $\xi_{Q}(q)$.

Definition 2.1 Let $\tau$ be a Lie algebra valued horizontal one form on $Q$; that is, a one form with values in the Lie algebra $\mathfrak{g}$ of $G$ that annihilates vertical vectors. The $\tau$-horizontal space at $q \in Q$ consists of tangent vectors to $Q$ at $q$ of the form $\operatorname{Hor}_{\tau} v_{q}=\operatorname{Hor} v_{q}-$ $[\tau(v)]_{Q}(q)$, which also defines $v_{q} \mapsto \operatorname{Hor}_{\tau}\left(v_{q}\right)$, called the $\tau$-horizontal projection. The $\tau$-vertical projection operator is defined by $\operatorname{Ver}_{\tau}\left(v_{q}\right):=\operatorname{Ver}\left(v_{q}\right)+[\tau(v)]_{Q}(q)$.

Definition 2.2 Given $g_{\sigma}, g_{\rho}$ and $\tau$, the controlled Lagrangian is the following:

$$
\begin{aligned}
L_{\tau, \sigma, \rho}(v) & =\frac{1}{2}\left[g_{\sigma}\left(\operatorname{Hor}_{\tau} v_{q}, \operatorname{Hor}_{\tau} v_{q}\right)\right. \\
& \left.+g_{\rho}\left(\operatorname{Ver}_{\tau} v_{q}, \operatorname{Ver}_{\tau} v_{q}\right)\right]-V(q) .
\end{aligned}
$$

The equations corresponding to this Lagrangian will be our closed-loop equations. The new terms appearing in those equations corresponding to the directly controlled variables are interpreted as control inputs. The modifications to the Lagrangian are chosen so that no new terms appear in the equations corresponding to the variables that are not directly controlled. We refer to this process as matching.

Once the control law is derived using the controlled Lagrangian, the closed-loop stability of an equilibrium can be determined by energy methods, using any available freedom in the choice of $\tau, g_{\sigma}$ and $g_{\rho}$.

Under some reasonable assumptions on the metric $g_{\sigma}, L_{\tau, \sigma, \rho}(v)$ has the following useful structure.

Theorem 2.3 Assume that $g=g_{\sigma}$ on Hor and Hor and Ver are orthogonal for $g_{\sigma}$. Then

$L_{\tau, \sigma, \rho}(v)=L\left(v+\tau(v)_{Q}\right)+\frac{1}{2} g_{\sigma}\left(\tau(v)_{Q}, \tau(v)_{Q}\right)+\frac{1}{2} \varpi(v)$ where $v \in T_{q} Q$ and $\varpi(v)=\left(g_{\rho}-g\right)\left(\operatorname{Ver}_{\tau}(v), \operatorname{Ver}_{\tau}(v)\right)$. 
The coordinate formula for $L$ is

$$
L=\frac{1}{2} g_{\alpha \beta} \dot{x}^{\alpha} \dot{x}^{\beta}+g_{\alpha a} \dot{x}^{\alpha} \dot{\theta}^{a}+\frac{1}{2} g_{a b} \dot{\theta}^{a} \dot{\theta}^{b}-V\left(x^{\alpha}\right) .
$$

and coordinate formula for $L_{\tau, \sigma, \rho}$ is

$$
\begin{aligned}
& L_{\tau, \sigma, \rho}(v)=L\left(x^{\alpha}, \dot{x}^{\beta}, \dot{\theta}^{a}+\tau_{\alpha}^{a} \dot{x}^{\alpha}\right)+\frac{1}{2} \sigma_{a b} \tau_{\alpha}^{a} \tau_{\beta}^{b} \dot{x}^{\alpha} \dot{x}^{\beta} \\
& \quad+\frac{1}{2} \varpi_{a b}\left(\dot{\theta}^{a}+g^{a c} g_{\alpha c} \dot{x}^{\alpha}+\tau_{\alpha}^{a} \dot{x}^{\alpha}\right)\left(\dot{\theta}^{b}+g^{b d} g_{\beta d} \dot{x}^{\beta}+\tau_{\beta}^{b} \dot{x}^{\beta}\right) .
\end{aligned}
$$

Here, $\theta^{a}$ are coordinates for the abelian symmetry group $G$ and $x^{\alpha}$ are coordinates on the shape space $Q / G ; \sigma_{a b}$ and $\varpi_{a b}$ are the coefficients for the last two terms, respectively, of the expression for $L_{\tau, \sigma, \rho}$ in Theorem 2.3, and we let $\rho_{a b}=g_{a b}+\varpi_{a b}$. The associated controlled conserved quantity is given by

$$
\tilde{J}_{a}:=\frac{\partial L_{\tau, \sigma, \rho}}{\partial \dot{\theta}^{a}}=\rho_{a b}\left(\dot{\theta}^{b}+g^{b d} g_{\alpha d} \dot{x}^{\alpha}+\tau_{\alpha}^{b} \dot{x}^{\alpha}\right) .
$$

General sufficient conditions for matching can be found in Bloch, Leonard and Marsden [1999b]. We define simplified sufficient conditions for matching that are satisfied for a class of systems that includes the inverted (planar or spherical) pendulum on a cart. We take $g_{\rho}=g$ and the simplified matching conditions are

SM-1 $\sigma_{a b}=\sigma g_{a b}$ for a constant $\sigma$ (this defines $\sigma_{a b}$ ),

SM-2 $g_{a b}$ is independent of $x^{\alpha}$ (a condition on the metric tensor),

SM-3 $\tau_{\alpha}^{b}=-(1 / \sigma) g^{a b} g_{\alpha a}$ (this defines $\left.\tau_{\alpha}^{b}\right)$,

SM-4 $g_{\alpha a, \delta}=g_{\delta a, \alpha}$ (a second condition on the metric).

We use commas to denote partial differentiation with respect to $x^{\alpha}$. The conditions SM-2 and SM-4 imply that the mechanical connection $g^{a b} g_{a \alpha}$ for the given system is flat, i.e., systems that satisfy the simplified matching conditions lack gyroscopic forces.

Define $\kappa=-1 / \sigma$. Under the simplified matching assumptions SM-1 - SM-4, the control law is computed to be $u_{a}=-d / d t\left(\kappa g_{\alpha a} \dot{x}^{\alpha}\right)$. Acceleration terms can be eliminated such that the control law becomes

$$
\begin{aligned}
u_{a}= & -\kappa\left\{g_{\beta a, \gamma}-g_{\delta a} A^{\delta \alpha}\left[g_{\alpha \beta, \gamma}-\frac{1}{2} g_{\beta \gamma, \alpha}\right.\right. \\
& \left.\left.-(1+\kappa) g_{\alpha d} g^{d a} g_{\beta a, \gamma}\right]\right\} \dot{x}^{\beta} \dot{x}^{\gamma} \\
& +\kappa g_{\delta a} A^{\delta \alpha} \frac{\partial V}{\partial x^{\alpha}}
\end{aligned}
$$

where $A_{\alpha \beta}=g_{\alpha \beta}-g_{\alpha d}(1+\kappa) g^{d a} g_{\beta a}$.

An equilibrium for the controlled system corresponds to $x_{e}^{\alpha}, \dot{x}_{e}^{\alpha}=0$ and $\tilde{J}_{a}=\mu_{a}$. Let

$$
V_{\mu}\left(x^{\alpha}\right)=V\left(x^{\alpha}\right)+\frac{1}{2} g^{a b} \mu_{a} \mu_{b}
$$

Theorem 2.4 Assume SM-1 - SM-4 hold. Then, the given equilibrium is stabilized by the control law (2.6) if the second variation of

$$
E_{\mu}:=\frac{1}{2} A_{\alpha \beta} \dot{x}^{\alpha} \dot{x}^{\beta}+V_{\mu}
$$

(as a function of the variables $x^{\alpha}$ ) evaluated at the equilibrium is definite.

Suppose $x_{e}^{\alpha}$ is a maximum of $V_{\mu}$. Then we would need to make the equilibrium a maximum of $E_{\mu}$. If, for example, $g_{a \alpha}\left(x_{e}^{\alpha}\right)$ is one-to-one, then $A_{\alpha \beta}$ will be negative definite for a choice of $\kappa$ such that

$1+\kappa>\max \left\{\lambda \mid \operatorname{det}\left(g_{\alpha \beta}\left(x_{e}\right)-\lambda g_{\alpha a}\left(x_{e}\right) g^{a b} g_{b \beta}\left(x_{e}\right)\right)=0\right\}$.

See Bloch, Chang, Leonard and Marsden [1999] for details.

\section{Matching with Symmetry-Breaking Potentials}

In this section we extend the method of controlled Lagrangians to the class of Lagrangian mechanical systems with potential energy that may break symmetry, i.e., we still have a symmetry group $G$ for the kinetic energy of the system but we now have a potential energy of the form $V=V\left(x^{\alpha}, \theta^{a}\right)$ that need not be $G$ invariant. Further, we consider a modification to the potential energy that also breaks symmetry in the $G$ variables. Let the potential energy for the controlled Lagrangian $V^{\prime}$ be defined as

$$
V^{\prime}\left(x^{\alpha}, \theta^{a}\right)=V\left(x^{\alpha}, \theta^{a}\right)+V_{\epsilon}\left(x^{\alpha}, \theta^{a}\right)
$$

where $V_{\epsilon}$ is the modification to be determined.

We specialize to the case of mechanical systems for which the simplified matching assumptions SM-1 - SM4 hold. However, we retain the flexibility afforded by $g_{\rho}$. We consider $\rho_{a b}=\rho g_{a b}$ where $\rho$ is a scalar constant. The controlled Lagrangian takes the form

$$
\begin{aligned}
& L_{\tau, \sigma, \rho, \epsilon}(v)=L\left(x^{\alpha}, \dot{x}^{\beta}, \dot{\theta}^{a}+\tau_{\alpha}^{a} \dot{x}^{\alpha}\right)+\frac{1}{2} \sigma g_{a b} \tau_{\alpha}^{a} \tau_{\beta}^{b} \dot{x}^{\alpha} \dot{x}^{\beta} \\
+ & \frac{1}{2}(\rho-1) g_{a b}\left(\dot{\theta}^{a}+g^{a c} g_{\alpha c} \dot{x}^{\alpha}+\tau_{\alpha}^{a} \dot{x}^{\alpha}\right) \\
& \left(\dot{\theta}^{b}+g^{b d} g_{\beta d} \dot{x}^{\beta}+\tau_{\beta}^{b} \dot{x}^{\beta}\right)-V_{\epsilon}\left(x^{\alpha}, \theta^{a}\right),
\end{aligned}
$$

The conjugate momenta $\tilde{J}_{a}$ to $\theta^{a}$ then becomes

$$
\tilde{J}_{a}=\frac{\partial L_{\tau, \sigma, \rho, \epsilon}}{\partial \dot{\theta}^{a}}=\rho g_{a b}\left(\dot{\theta}^{b}+g^{b d} g_{\alpha d} \dot{x}^{\alpha}+\tau_{\alpha}^{b} \dot{x}^{\alpha}\right) .
$$

The new Euler-Lagrange equations in the $\theta^{a}$ variables become

$$
\frac{d}{d t}\left(\frac{\partial L_{\tau, \sigma, \rho, \epsilon}}{\partial \dot{\theta}^{a}}\right)+\frac{\partial V}{\partial \theta^{a}}+\frac{\partial V_{\epsilon}}{\partial \theta^{a}}=0 .
$$


Comparing this equation to our controlled $\theta^{a}$ equation

$$
\frac{d}{d t}\left(\frac{\partial L}{\partial \dot{\theta}^{a}}\right)+\frac{\partial V}{\partial \theta^{a}}=u_{a},
$$

the control law can be read off as

$$
\begin{aligned}
u_{a} & =\frac{d}{d t}\left(\frac{\partial L}{\partial \dot{\theta}^{a}}-\frac{1}{\rho} \frac{\partial L_{\tau, \sigma, \rho, \epsilon}}{\partial \dot{\theta}^{a}}\right)+\frac{\rho-1}{\rho} \frac{\partial V}{\partial \theta^{a}}-\frac{1}{\rho} \frac{\partial V_{\epsilon}}{\partial \theta^{a}} \\
& =-\frac{d}{d t}\left(g_{a b} \tau_{\alpha}^{b} \dot{x}^{\alpha}\right)+\frac{\rho-1}{\rho} \frac{\partial V}{\partial \theta^{a}}-\frac{1}{\rho} \frac{\partial V_{\epsilon}}{\partial \theta^{a}}
\end{aligned}
$$

The next step is to determine conditions so that the Euler-Lagrange equations in the $x^{\alpha}$ variables still match. Our job is to find conditions such that

$$
\mathcal{E}_{x}\left(L_{\tau, \sigma, \rho, \epsilon}\right):=\frac{d}{d t} \frac{\partial L_{\tau, \sigma, \rho, \epsilon}}{\partial \dot{x}^{\alpha}}-\frac{\partial L_{\tau, \sigma, \rho, \epsilon}}{\partial x^{\alpha}}=0 .
$$

Define $L_{\tau, \sigma}$ as the controlled Lagrangian $L_{\tau, \sigma, \rho}$ in the case that $g_{\rho}=g$. Then, using the simplified matching assumptions

$$
\mathcal{E}_{x}\left(L_{\tau, \sigma, \rho, \epsilon}\right)=\mathcal{E}_{x}\left(L_{\tau, \sigma}\right)+\frac{\rho-1}{\rho}\left(g^{b d} g_{\alpha d}+\tau_{\alpha}^{b}\right) \dot{\tilde{J}}_{b} .
$$

Using the calculation of $\mathcal{E}_{x}\left(L_{\tau, \sigma}\right)$ from Bloch, Leonard and Marsden [1999a], we compute

$$
\begin{aligned}
\mathcal{E}_{x}\left(L_{\tau, \sigma, \rho, \epsilon}\right) & =\frac{1}{\rho} \dot{\bar{J}}_{a} \tau_{\alpha}^{a}+\frac{\partial V_{\epsilon}}{\partial x^{\alpha}}+\frac{\rho-1}{\rho}\left(g^{a d} g_{\alpha d}+\tau_{\alpha}^{a}\right) \dot{\bar{J}}_{a} \\
& =-\frac{\partial V^{\prime}}{\partial \theta^{a}}\left(-\frac{1}{\sigma}+\frac{\rho-1}{\rho}\right) g^{a d} g_{\alpha d}+\frac{\partial V_{\epsilon}}{\partial x^{\alpha}} .
\end{aligned}
$$

We define a new matching condition:

\section{Assumption SM-5:}

$$
-\left(\frac{\partial V}{\partial \theta^{a}}+\frac{\partial V_{\epsilon}}{\partial \theta^{a}}\right)\left(-\frac{1}{\sigma}+\frac{\rho-1}{\rho}\right) g^{a d} g_{\alpha d}+\frac{\partial V_{\epsilon}}{\partial x^{\alpha}}=0 .
$$

A necessary and sufficient condition on $V$ and $g^{a b} g_{a \alpha}$ for the existence of $V_{\epsilon}$ satisfying SM-5 is given and proved in Bloch, Chang, Leonard and Marsden [1999]. The following theorem gives sufficient conditions for matching with symmetry-breaking potentials.

Theorem 3.1 (Matching with Potential Shaping) Under Assumptions SM-1, SM-2, SM-3, SM-4, SM5 the Euler-Lagrange equations for the controlled Lagrangian $L_{\tau, \sigma, \rho, \epsilon}$ coincide with the controlled Euler-Lagrange equations.

\section{Stabilization with Symmetry Breaking Potentials}

In the case that the conditions for Theorem 3.1 are satisfied, the energy $E_{\tau, \sigma, \rho, \epsilon}$ associated to the closedlonp system can be used as a Lyapunov function. In particular, we use it to assign the remaining freedom in $\sigma, \rho$ and $\epsilon$ to guarantee stability of an equilibrium of interest. Any equilibrium will have the form, $\left(x^{\alpha}, \theta^{a}, \dot{x}^{\alpha}, \dot{\theta}^{a}\right)=\left(x_{e}^{\alpha}, \theta_{e}^{a}, 0,0\right)$.

We compute $E_{\tau, \sigma, \rho, \epsilon}$ to be

$$
\begin{aligned}
E_{\tau, \sigma, \rho, \epsilon} & =\frac{\partial L_{\tau, \sigma, \rho, \epsilon}}{\partial \dot{x}^{\alpha}} \dot{x}^{\alpha}+\frac{\partial L_{\tau, \sigma, \rho, \epsilon}}{\partial \dot{\theta}^{a}} \dot{\theta}^{a}-L_{\tau, \sigma, \rho, \epsilon} \\
& =\frac{1}{2} A_{\alpha \beta} \dot{x}^{\alpha} \dot{x}^{\beta}+\frac{1}{2} \rho g_{a b} \zeta^{a} \zeta^{b}+V^{\prime}\left(x^{\alpha}, \theta^{a}\right),
\end{aligned}
$$

where $\zeta^{a}=\dot{\theta}^{a}+(1-1 / \sigma) g^{a b} g_{\alpha b} \dot{x}^{\alpha}$.

The Lagrange-Dirichlet Theorem then gives the following sufficient conditions for Lyapunov stability.

Theorem 4.1 (Lyapunov Stability) Assume SM-1 - SM-5 hold. The equilibrium defined by $\left(x_{e}^{\alpha}, \theta_{e}^{a}, 0,0\right)$ is Lyapunov stable if it is a critical point of $V^{\prime}$ and if the second derivative of $E_{\tau, \sigma, \rho, \epsilon}$ evaluated at the equilibrium is definite.

Note that if $V_{\epsilon}$ is chosen to make $\left(x_{e}^{\alpha}, \theta_{e}^{a}\right)$ a maximum of $V^{\prime}\left(x^{\alpha}, \theta^{a}\right)$, then choosing $\kappa$ to satisfy (2.9) and $\rho<0$ is sufficient for stability.

For asymptotic stability, we will want to add in a dissipative control term, i.e.,

$$
u_{a}=u_{a}^{\mathrm{cons}}+\frac{1}{\rho} u_{a}^{\mathrm{diss}}
$$

where from (3.2)

$$
u_{a}^{\text {cons }}=-\frac{d}{d t}\left(g_{a b} \tau_{\alpha}^{b} \dot{x}^{\alpha}\right)+\frac{\rho-1}{\rho} \frac{\partial V}{\partial \theta^{a}}-\frac{1}{\rho} \frac{\partial V_{\epsilon}}{\partial \theta^{a}} .
$$

The Euler-Lagrange equations in terms of the controlled Lagrangian then become

$$
\begin{aligned}
& \frac{d}{d t} \frac{\partial L_{\tau, \sigma, \rho, \epsilon}}{\partial \dot{x}^{\alpha}}-\frac{\partial L_{\tau, \sigma, \rho, \epsilon}}{\partial x^{\alpha}}=\left(-\frac{1}{\sigma}+\frac{\rho-1}{\rho}\right) g^{a d} g_{\alpha d} u_{a}^{\text {diss }} \\
& \frac{d}{d t} \frac{\partial L_{\tau, \sigma, \rho, \epsilon}}{\partial \dot{\theta}^{a}}-\frac{\partial L_{\tau, \sigma, \rho, \epsilon}}{\partial \dot{\theta}^{a}}=u_{a}^{\text {diss }}
\end{aligned}
$$

Thus, one can compute that

$$
\begin{gathered}
\frac{d}{d t} E_{\tau, \sigma, \rho, \epsilon}=\xi^{a} u_{a}^{\mathrm{diss}}, \\
\xi^{a}=\dot{\theta}^{a}+\left(-\frac{1}{\sigma}+\frac{\rho-1}{\rho}\right) g^{a d} g_{\alpha d} \dot{x}^{\alpha} .
\end{gathered}
$$

Therefore, we can choose

$$
u_{a}^{\text {diss }}=c_{a}^{d} g_{b d} \xi^{b}
$$

where $c_{a}^{d}$ is a positive (negative) definite control gain matrix if the equilibrium is a maximum (minimum) of $\left.E_{\tau, \sigma, \rho, \epsilon}\right)$. This gives $\frac{d}{d t} E_{\tau, \sigma, \beta, \epsilon}=c_{a}^{d} g_{b d} \xi^{a} \xi^{b}$.

In order to get asymptotic stability of the equilibrium, we use LaSalle's invariance principle. From above, we see that $d / d t\left(E_{\tau, \sigma, \rho, \epsilon}\right)$ vanishes on the set $\mathcal{M}$ defined by $u_{a}^{\text {diss }}=c_{a}^{d} 1 / \rho\left(\tilde{J}_{d}-g_{\alpha d} \dot{x}^{\alpha}\right)=0$. 
Theorem 4.2 (Asymptotic Stabilization) Assume that the hypotheses of the Stabilization Theorem 4.1 as well as the assumptions SM-1 SM-5 hold. In addition, assume that $\mathcal{M}$ consists only of equilibria and that the dissipative control law is chosen as in (4.4). Then the given equilibrium is asymptotically stable.

Specific conditions under which these hypotheses can be verified are investigated in Bloch, Chang, Leonard and Marsden [1999].

We again define $\kappa=-1 / \sigma$. The total control $u_{a}$ is

$$
u_{a}=u_{a}^{\text {cons }}+\frac{1}{\rho} u_{a}^{\text {diss }}=-\frac{d}{d t}\left(\kappa g_{\alpha a} \dot{x}^{\alpha}\right)+w_{a}
$$

where

$$
w_{a}=\frac{\rho-1}{\rho} \frac{\partial V}{\partial \theta^{a}}-\frac{1}{\rho} \frac{\partial V_{\epsilon}}{\partial \theta^{a}}+\frac{1}{\rho} u_{a}^{\mathrm{diss}}
$$

This control law is the sum of our original stabilizing control law without symmetry breaking plus the potential modification and the dissipation term.

Following the same procedure as in Bloch, Leonard and Marsden [1999a], we can eliminate accelerations in the control law expression. We compute

$u_{a}=(\operatorname{rhs}$ of $(2.7))+\kappa g_{\delta a} A^{\delta \alpha} \frac{1}{\rho} g_{\alpha d} g^{d b}\left(-\frac{\partial V^{\prime}}{\partial \theta^{b}}+u_{b}^{\text {diss }}\right)+w_{a} \cdot$

\section{Inverted Pendulum on an Inclined Cart}

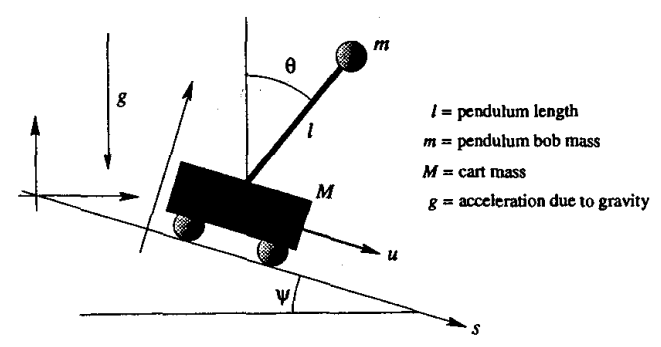

Figure 5.1: The pendulum on an inclined cart.

We apply the above result to stabilize the inverted planar pendulum on a cart that travels on an incline of angle $\psi$. Let $s$ denote the position of the cart along the incline and let $\theta$ denote the angle of the pendulum with the upright vertical as shown in Figure 5.1.

The configuration space for this system is $Q=$ $S \times G=S^{1} \times \mathbb{R}$, with the first factor being the pendulum angle $\theta$ and the second factor being the cart position $s$. The velocity phase space $T Q$ has coordinates $z=(\theta, s, \dot{\theta}, \dot{s})$. We are interested in the problem of asymptotically stabilizing the origin, i.e., $z=0$.
The velocity of the cart relative to the lab frame is $\dot{s}$, while the velocity of the pendulum relative to the lab frame is the vector

$$
v_{\text {pend }}=(\dot{s} \cos \psi+l \cos \theta \dot{\theta},-\dot{s} \sin \psi-l \sin \theta \dot{\theta}) .
$$

The system kinetic energy $K(\theta, s, \dot{\theta}, \dot{s})$ is the sum of the kinetic energies of the cart and the pendulum:

$$
\frac{1}{2}[\dot{\theta}, \dot{s}]\left[\begin{array}{cc}
m l^{2} & m l \cos (\theta-\psi) \\
m l \cos (\theta-\psi) & M+m
\end{array}\right]\left[\begin{array}{c}
\dot{\theta} \\
\dot{s}
\end{array}\right] .
$$

The potential energy is

$$
V(\theta, s)=m g l \cos \theta-(m+M) g s \sin \psi .
$$

The Lagrangian is the kinetic minus potential energy:

$$
\begin{gathered}
L(\theta, s, \dot{\theta}, \dot{s})=\frac{1}{2}\left(\alpha \dot{\theta}^{2}+2 \beta \cos (\theta-\psi) \dot{s} \dot{\theta}+\gamma \dot{s}^{2}\right) \\
+D \cos \theta+\gamma g s \sin \psi,
\end{gathered}
$$

where $\alpha=m l^{2}, \beta=m l, \gamma=M+m$ and $D=-m g l$ are constants. Notice that the potential energy breaks symmetry in the cart translation $s$ (although this system is still translation invariant).

The equations of motion for the cart pendulum system with a control force $u$ acting on the cart (and no direct forces acting on the pendulum) are

$$
\begin{aligned}
& \frac{d}{d t} \frac{\partial L}{\partial \dot{\theta}}-\frac{\partial L}{\partial \theta}=0 \\
& \frac{d}{d t} \frac{\partial L}{\partial \dot{s}}-\frac{\partial L}{\partial s}=u .
\end{aligned}
$$

By inspection we see that SM-2 and SM-4 hold. To satisfy SM-1 and SM-3, we take $\sigma_{a b}=\sigma g_{a b}=\sigma \gamma$ and $\tau_{\alpha}^{b}=-(1 / \sigma) g^{a b} g_{\alpha a}=(\kappa / \gamma) \beta \cos (\theta-\psi)$, where $\sigma$ is a scalar constant and $\kappa=-1 / \sigma$.

We choose $V_{\epsilon}$ to be $V_{\epsilon}=\tilde{V}_{\epsilon}+\gamma g s \sin \psi$,

where

$\tilde{V}_{\epsilon}=\frac{1}{2} \epsilon D \frac{\gamma^{2}}{\beta^{2}}\left(s+\left(\kappa+\frac{\rho-1}{\rho}\right) \frac{\beta}{\gamma}(\sin (\theta-\psi)+\sin \psi)\right)^{2}$.

It is easy to check that with this definition, SM-5 holds. Thus, by Theorem 3.1 we have matching with potential shaping.

This means that if we apply the control law defined by (3.2), the closed-loop system is Lagrangian and the associated energy as given by (4.1) is

$$
E_{\tau, \sigma, \rho, \epsilon}=\frac{1}{2} A \dot{\theta}^{2}+\frac{1}{2} \frac{\rho}{\gamma}\left(\dot{s}+(1+\kappa) \frac{\beta}{\gamma} \cos (\theta-\psi) \dot{\theta}^{2}\right)^{2}+V^{\prime}
$$

where $V^{\prime}=-D \cos \theta+\tilde{V}_{\epsilon}$ and

$$
A=\alpha-(1+\kappa) \beta^{2} / \gamma \cos ^{2}(\theta-\psi) .
$$


The point $z=0$ is an equilibrium of the controlled Lagrangian system since it is a critical point of $V^{\prime}$. It remains to find conditions on $\kappa, \rho, \epsilon$ such that the second derivative of $E_{\tau, \sigma, \rho, \epsilon}$ evaluated at the origin is definite. First note that if we take $\epsilon>0$, then $(\theta, s)=(0,0)$ is a maximum of the potential energy $V^{\prime}$. Further, by (2.9) $A$ is negative definite if

$$
\kappa>\frac{\alpha \gamma}{\beta^{2} \cos ^{2} \psi}-1=\frac{m \sin ^{2} \psi+M}{m \cos ^{2} \psi} .
$$

Finally, we take $\rho<0$ so that the second derivative of $E_{\tau, \sigma, \rho, \epsilon}$ evaluated at the origin is negative definite. Thus, by Theorem 4.1, the origin is made Lyapunov stable by the control law given by (3.2) (and (4.6) with $u^{\text {diss }}=0$ ).

We can further get asymptotic stability using the full control law given by (4.6) where we choose $c_{a}^{d}=$ $c>0$ since the equilibrium is a maximum of $E_{\tau, \sigma, \rho, \epsilon}$. The dissipation term is

$$
u^{\mathrm{diss}}=c \gamma\left(\dot{s}+\left(\kappa+\frac{\rho-1}{\rho}\right) \frac{\beta}{\gamma} \cos (\theta-\psi) \dot{\theta}\right) .
$$

The complete control law (4.6) becomes

$$
\begin{aligned}
u= & \left.\frac{1}{A}\left(\kappa \beta\left(\alpha \sin (\theta-\psi) \dot{\theta}^{2}+\cos (\theta-\psi) D \sin \theta\right)\right)\right) \\
& +\frac{1}{A}\left(B \frac{\partial V^{\prime}}{\partial s}+C u^{\text {diss }}\right)-\gamma g \sin \psi,
\end{aligned}
$$

where $B=-1 / \rho\left(\alpha-\beta^{2} / \gamma \cos ^{2}(\theta-\psi)\right)$ and $C=\alpha-$ $(\kappa+1-\kappa / \rho) \beta^{2} / \gamma \cos ^{2}(\theta-\psi)$.

This control law is finite if the denominator is strictly negative, i.e., if

$$
\sin ^{2}(\theta-\psi)<\frac{\beta^{2}(\kappa+1)-\alpha \gamma}{\beta^{2}(\kappa+1)} .
$$

This range of $\theta$ tends to the range $-(\pi / 2+\psi)<\theta<$ $\pi / 2+\psi$ for large $\kappa$. Asymptotic stability follows by showing that the set $\mathcal{M}$, which is equivalent to the set on which $\frac{\partial \tilde{V}_{e}}{\partial s}$ is constant, contains only equilibria. See Bloch, Chang, Leonard and Marsden [1999] for the proof, for an investigation of the region of attraction, and for simulation results.

\section{References}

Åström, K.J. and K. Furuta [1996] Swinging up a pendulum by energy control. IFAC, San Francisco 13.

Auckly, D., L. Kapitanski and W. White [1998] Control of nonlinear underactuated systems. Preprint.

Baillieul, J. [1993] Stable average motions of mechanical systems subject to periodic forcing. Fields Inst. Comm. 1, 1-23.
Bloch, A.M., D.E. Chang, N.E. Leonard and J.E. Marsden [1999] Controlled Lagrangians and the stabilization of mechanical systems II: Potential shaping and tracking. Preprint.

Bloch, A.M., P.S. Krishnaprasad, J.E. Marsden and G. Sánchez de Alvarez [1992] Stabilization of rigid body dynamics by internal and external torques. Automatica 28, 745-756.

Bloch, A.M., N.E. Leonard and J.E. Marsden [1997] Stabilization of mechanical systems using controlled Lagrangians, Proc CDC 36, 2356-2361.

Bloch, A.M., N.E. Leonard and J.E. Marsden [1998] Matching and stabilization by the method of controlled Lagrangians, Proc CDC 37, 1446-1451.

Bloch, A.M., N.E. Leonard and J.E. Marsden [1999a] Controlled Lagrangians and the stabilization of mechanical systems I: The first matching theorem, IEEE Trans. Aut. Control. To appear.

Bloch, A.M., N.E. Leonard and J.E. Marsden [1999b] Stabilization of the pendulum on a rotor arm by the method of controlled Lagrangians, Proc. of ICRA ' 99 , IEEE, 500-505.

Bloch, A.M., J.E. Marsden, G. Sánchez de Alvarez [1997] Stabilization of relative equilibria of mechanical systems with symmetry. Proc. Notre Dame Conf. on Applied Mathematics, Birkhäuser.

Hamberg, J. [1999] General matching conditions in the theory of controlled Lagrangians. Preprint.

Krishnaprasad, P.S. [1985] Lie-Poisson structures, dual-spin spacecraft and asymptotic stability, Nonl. Anal. Th. Meth. and Appl. 9, 1011-1035.

Leonard, N.E. [1997] Stabilization of underwater vehicle dynamics with symmetry-breaking potentials, Systems and Control Letters 32, 35-42.

Marsden, J.E. [1992], Lectures on Mechanics London Mathematical Society Lecture note series. 174, Cambridge University Press.

Marsden, J.E. and T.S. Ratiu [1994] Introduction to Mechanics and Symmetry. Texts in Applied Mathematics, 17, Springer-Verlag. Second Edition, 1999.

van der Schaft, A. J. [1986], Stabilization of Hamiltonian systems, Nonlinear Analysis, Theory, Methods and Applications, 10, 1021-1035.

Wang, L.S. and P.S. Krishnaprasad [1992] Gyroscopic control and stabilization, J. Nonlinear Sci. 2 , $367-415$. 